Ambient Science, 2020: Vol. 07(Sp1); 30-32

DOI:10.21276/ambi.2020.07.sp1.oa03

\title{
Social Appearance Anxiety of University Students from Urban and Rural Backgrounds
}

\author{
Abdullah Bora Özkara \\ Turkey \\ Study Area: Erzurum, Turkey \\ Coordinates: $39^{\circ} 54^{\prime} 31$ ? N $41^{\circ} 16^{\prime} 37$ ? E
}

Faculty of Sport Sciences, Erzurum Technical University, Erzurum,

Key words: Urban Vs rural life, Sport participation

\section{Abstract}

The aim of this study is to examine the social appearance anxiety of university students who grew up in urban and rural areas. Personal information form and "Social Appearance Anxiety Scale (SAAS)" were used as data collection tools. As a statistical method in the evaluation of the data obtained; descriptive statistics (kurtosis and skewness) and t-test analysis for independent groups were used. No significant differences were detected in terms of variables of gender and residence (urban and rural Backgrounds). However, a signif icant difference was found in favor of those who stated that university students participated in sports in terms of the variable of participation in sports. Although no significant difference is identified, this research has revealed that urban and rural youth have social appearance anxiety, but combating this anxiety is a variable that makes a difference.

Another situation faced by young people during the university period is their cultural differences. Popular culture makes itself feel excessive in cities today. The characteristics of the families of university students and the geography they grow up in are different from each other. The foundations of the social culture of the young people with family growing up in the city living in the city and the young people who have family roots in the rural areas may be different from each other. Interaction of young people with different cultures is supported by their tendency to accept and not see themselves or their peers differently, but this is not a problem. Because urban life and rural life are phenomena that feed each other. Coexistence of young people from two different backgrounds can be considered as a cultural wealth (Aksoy, 2016; Yahyagil, 1998; Zu, 2006).

A university degree in Turkey in recent years has shown a substantial increase. The new universities established in the cities after the 2000 s have given young people opportunities to establish their social and cultural relations in a healthier way. In this process, with the increasing number of university students, young people who are in the life lived on campuses and the life lived outside the campus have started to give more importance to people's perceptions of their appearance. The state of anxiety experienced by young people because they are afraid of the evaluations of others, whose opinions they care about, has an important stimulating feature in terms of activating them. Young people have started to attach more importance

*Author: bora.ozkara@erzurum.edu.tr 
to exercise and physical activity because of this anxiety about their opinions and their sensitivity to healthy life. It is known that sports are the subject of health sciences, social sciences and performance research (Çelik, 2020; Özbay et al., 2018; Kirkbir, 2020). It can be said that other interventions other than sports and physical activity are encountered with aesthetic concerns. Of course, this process may have led to different or similar experiences for young people growing up in urban and rural areas. Hence, the aim of this study is to examine the social appearance concerns of university students in terms of some variables according to the geographical unit (urban and rural) they grew up in.

\section{Materials and Methods:}

The survey method, which tries to describe the views of the group, was used in the studies conducted on large groups of people included in the descriptive research method (Karasar, 2009). The data were collected online by the researcher. Participation was made on the basis of voluntary participation in the questionnaire form sent online to students' e-mail addresses. Ensuring the conf identiality of the data was given to the students by providing the necessary information.

The research consists of a sample group of university students studying in Erzurum and Trabzon in the spring semester in the 2017-2018 academic year. The usable responses of the students to whom the online data collection form was submitted were included in the evaluation. Accordingly, 371 (16o females, 211 males; age = 22.29) university students were the sample groups.

Data Collection Tools: personal information form created by the researcher and the Social Appearance Anxiety scale developed by Hart et al. (2008) and whose Turkish validity and reliability study was conducted by Dogan (2010) were used as data collection tools. Questions in the personal information form; It consists of variables of gender, place of residence, academic achievement and age. The social appearance anxiety scale consists of 16 items. It has a 5-Likert type answer key such as (1) Not Available at all, (5) Fully Available. The first item of the scale is coded in reverse. High scores from SSI, which measures social appearance anxiety in one dimension, indicate that appearance anxiety is high.

Data Analyzes: frequency, arithmetic mean standard deviation, kurtosis and skewness values among the descriptive statistics were used. For the differences between groups, t-Test analyzes were used for independent groups.

\section{Results:}

The kurtosis, skewness and standard deviation values of the data obtained from the social appearance anxiety scale form from 371 students, 160 of whom were women participating in the study, are shown in Table 1.
Table-1: Demographic information of university students

\begin{tabular}{llllll}
\hline Gender & $\mathrm{n}$ & $(\%)$ & $\mathrm{Sd}$ & Skewness & Kurtosis \\
\hline Female & 160 & 43.1 & 11.04 & 1.2 & 2.09 \\
Male & 211 & 56.9 & 10.11 & 0.79 & 0.15 \\
\hline
\end{tabular}

According to the results of the t-Test analysis conducted to evaluate the social appearance anxiety of the students in terms of the gender variable, no significant difference was observed between the scores of male and female students on the scale (Table-2).

Table 2. T-test Results of SAA Scores according to Gender

\begin{tabular}{lllllll}
\hline SAAS & $\mathrm{N}$ & Ort. & SD & df & T & P \\
\hline Female & 160 & 25.49 & 11.04 & 368 & -1.228 & 0.44 \\
Male & 211 & 26.84 & 10.11 & & & \\
\hline
\end{tabular}

According to the results of the $\mathrm{t}$-Test analysis conducted to evaluate the social appearance anxiety of the students in terms of the variable of their place of residence, no significant difference was observed between students with urban and rural backgrounds (Table-3).

Table-3: T-test Results of SAA Scores according to background rural / urban status

\begin{tabular}{lllllll}
\hline Status & $\mathrm{N}$ & Ort. & $\mathrm{SD}$ & $\mathrm{df}$ & $\mathrm{T}$ & $\mathrm{P}$ \\
\hline Rural & 203 & 26,35 & 10,18 & 368 &,- 190 & 0.48 \\
Urban & 167 & 26.14 & 10.97 & & & \\
\hline
\end{tabular}

The results of the t-Test analysis conducted to examine the social appearance anxiety of students in terms of the variable of participation in sports with a license differ significantly, in favor of those who stated that they participated in sports with a license (Table-4).

Table-4: T-test Results of SAA Scores according to sports participation

\begin{tabular}{lllllll}
\hline Participation & $\mathrm{N}$ & Ort. & SD & $\mathrm{df}$ & $\mathrm{T}$ & $\mathrm{P}$ \\
\hline Yes & $\mathbf{1 5 4}$ & $\mathbf{2 4 , 4 8}$ & $\mathbf{9 , 3 1}$ & 368 & $\mathbf{- 2 , 7 6}$ & $\mathbf{0 . 0 1}$ \\
No & $\mathbf{2 1 4}$ & $\mathbf{2 7 . 5 3}$ & $\mathbf{1 1 , 1 9}$ & & & \\
\hline
\end{tabular}

\section{Discussion and Conclusion:}

When the results obtained in this study, in which the social appearance anxiety of university students were examined according to their background, was examined, it was seen that no significant difference was found in terms of the gender variable in the light of the data obtained from the students in the sample group of the study. Similarly, when the data obtained from the sample group in terms of the place of residence variable were analyzed, no significant difference was found in the data obtained from the social appearance apricot scale of university students who lived in urban and rural areas. The variable of doing sports with license, which was reached in this study and was seen to be the only significant difference, created a significant difference in favor of licensed sportsmen. In other words, the scores of the students who stated that they were interested in sports with a license, obtained from social 
appearance anxiety were signif icantly lower than the others.

When the results of the study were examined, it was also seen in different studies (Alemdag et al., 2016; Yüceant \& Ünlü, 2017; Ermis \& Imamoglu, 2019) that social opinion anxiety did not make a significant difference in the sample of university students. However, in the study conducted by Sanlier et al. (2018) where variables such as body mass index, healthy lifestyle behaviors and social appearance anxiety were examined, it was reported that men had significantly higher social appearance anxietyvalues.

When the research results are examined in terms of sports participation variable, the low level of apricot students participating in sports with a license is slightly consistent with the results presented in some studies (Alemdag et al., 2016; Atasoy et al., 2016). In another study (Hergüner et al., 2018) that investigated the social appearance anxiety of students participating in different sports branches, it was found that social appearance anxiety was significantly associated with sports participation. These results can be supported by the results of the current research.

It can be said that the results obtained in this study, in which the concerns of social views were examined in terms of the life background of university students, were not subject to many studies, especially in terms of the false location variable, therefore, since there was no significant difference in terms of urba and rural variables in this study, the results of the research are not supportable to different studies. The fact that the research was limited to the sample of university students and that it included the cities of Trabzon and Erzurum shows that the generalizability of the study is limited.

As can be understood from the results of this study, it can be emphasized that besides the known benefits of participating in sports, it can have positive effects on the anxiety of individuals, although it has been previously demonstrated. For researches that can be carried out after this, social appearance plot studies that include only larger sample groups in the sample of university students can be examined in terms of the place of residence. Up-to-date research on social appearance anxiety can be made, especially considering the restrictions experienced by students in the covid-19 period.

\section{References:}

Aksoy, Z. (2016): An exploration on students' self evaluations of intercultural sensitivity development in intercultural communication training. Selcuk Iletisim, 9 (3):34-53

Alemdag, S., Alemdag, C., \& Ozkara, A.B. (2016): Social appearance anxiety of fitness participants. Baltic J. Sport Health Sci., 3(102).
Atasoy, M., Karabulut, E.O., \& Yalçinkaya, A. (2016). Study on fear of negative evaluation, and social appearance anxiety of university students engaged in futsal. J. Phys. Edu. Sport Manag., $7(7): 50-55$.

Cash, T.F., \& Pruzinsky, T. (2002): Body image: A Handbook of Theory, Research and Clinical Practice. London: Guilford Press.

Çelik, A. (2020): A Systematic Review on Examination of ELearning Platforms in Sports Education. Af. Edu. Res. J., 8(2): 292-296.

Clark, D.M. \& Wells, A. (1995): A cognitive model of social phobia. Social phobia: Diagnosis, assessment and treatment pp. ,14359. In, Heimberg RG, Liebowitz MR, Hope DA, Schneier FR (eds.) Rumination And Negative Appraisal In Social Phobia. . Pub. by: Guilford Press, New York.

Dogan, T. (2010): Turkish adaptation of Social Appearance Anxiety Scale (SGKÖ): Validity and reliability study. Hacettepe Egitim Fakültesi Dergisi, 39:151-159.

Ermis, E., \& Imamoglu, G. (2019): The effects of sport education and fine arts education on social appearance anxiety. $L$. Edu. Train. Stud., 7(6): 1-6.

Hart, T.A., Flora, D.B., Palyo, S.A., Fresco, D.M., Holle, C., \& Heimberg, R.G. (2008): Development and examination of the Social Appearance Anxiety Scale. Assessment, 15,:48-59.

Hergüner, G., Turan, S., Yaman, Ç., \& Bayraktar, F.U. (2018): Determining the social appearance anxiety levels of the students participating in school sports in different branches. Acta Kinesiol., $12(1): 24-28$.

Karasar, N. (2009): Scientific Research Method: ConceptsPrinciples-techniques. Pub. by: Nobel Publication Distribution.

Kirkbir, F. (2020): Examining the relationship between healthy nutrition and happiness in athletes. L. Sports Edu., 4(2):125130.

Özbay, S., Ulupinar, S., \& Özkara, A. B. (2018): Agility Performance in Sports. L. Nat. Sport Sci., 2(2): 97-112.

Sanlier, N., Pehlivan, M., Sabuncular, G., Bakan, S., \& Isguzar, Y. (2018): Determining the relationship between body mass index, healthy lifestyle behaviors and social appearance anxiety. Ecol. Food Nutr., 57(2):124-139.

Yousefi, B., Hassani, Z., \& Shokri, O. (2009). Reliability and Factor Validity of the 7-Item Social Physique Anxiety Scale (SPAS- 7) among University Students in Iran. World J. Sport Sci., 2(3), 201-204.

Yahyagil, M.Y. (1998). The Effects of Cities on the Development of Culture. Sociology Conferences. Sosyoloji Konferanslari, (25), 105-120.

Yuceant, M., \& Unlu, H. (2017). The analysis of social appearance anxiety levels of physical education teacher candidates in terms of different variables. Turk. J. Sport Exerc., 19(1):102-108. 